\title{
Altered postural timing and abnormally low use of proprioception in lumbar spinal stenosis pre- and post- surgical decompression
}

\author{
Sarah Kneis ${ }^{1}$, Verena Bruetsch¹, Daniela Dalin', Ulrich Hubbe² and Christoph Maurer ${ }^{1 *}$
}

\begin{abstract}
Background: Lumbar spinal stenosis (LSS) is frequently associated with postural instability. Although several studies evaluated patients' functional impairments, underlying sensorimotor mechanisms are still poorly understood. We aimed to assess the specific set of postural control deficits associated with LSS during spontaneous and externally perturbed stance and evaluated post-surgical changes in postural behavior.

Methods: We analyzed postural control in eleven LSS patients (age $69 \pm 8$ years) pre- and post-laminectomy, correlated experimental data with functional tests and patient-reported outcomes, and compared findings to 15 matched, healthy control subjects (age $70 \pm 6$ years). Postural control was characterized by spontaneous sway measures and measures of perturbed stance. Perturbations were induced by anterior-posterior pseudorandom tilts of the body support surface. We used an established postural control model to extract specific postural control parameters.

Results: Spontaneous sway amplitude, velocity and frequency were abnormally large in LSS patients. Furthermore, patients' postural reactions to platform tilts, represented by GAIN and PHASE were significantly altered. Based on simple feedback model simulations, we found that patients rely less on proprioceptive cues for stance regulation than healthy subjects. Moreover, their postural reactions' timing is altered. After surgery, patients' spontaneous sway amplitude was significantly reduced and their postural timing approximated the behavior of healthy subjects.

Conclusion: The reduction in proprioceptive input for stance control due to stenosis-caused afferent dysfunction is a functional disadvantage for LSS patients - and may be the basis of increased spontaneous sway. This disadvantage may cause the timing of postural reactions to alter, with the intent of preventing rapid changes in stance regulation for safety reasons. After surgery, patients' postural timing approximated those of healthy subjects, while the abnormally low use of proprioception remained unchanged. We suggest the post-surgery rehabilitation of proprioception, eg through balance exercises on unstable surfaces and reduced visual input.
\end{abstract}

Keywords: Low back pain, Posturography, Postural balance, Center of pressure, Walking speed, Quality of life

\footnotetext{
* Correspondence: christoph.maurer@uniklinik-freiburg.de

${ }^{1}$ Department of Neurology, Faculty of Medicine and Medical Center -

University of Freiburg, Freiburg, Germany

Full list of author information is available at the end of the article
}

(c) The Author(s). 2019 Open Access This article is distributed under the terms of the Creative Commons Attribution 4.0 International License (http://creativecommons.org/licenses/by/4.0/), which permits unrestricted use, distribution, and reproduction in any medium, provided you give appropriate credit to the original author(s) and the source, provide a link to the Creative Commons license, and indicate if changes were made. The Creative Commons Public Domain Dedication waiver (http://creativecommons.org/publicdomain/zero/1.0/) applies to the data made available in this article, unless otherwise stated. 


\section{Background}

Degenerative lumbar spinal stenosis (LSS), which is commonly diagnosed between the 6 th and 7 th decade $[1,2]$, is often accompanied by functional impairments like gait and balance problems [3-9]. It is a frequent cause for spinal claudication and can lead to chronic or recurrent low back pain (LBP) syndrome [1, 2], which, in turn, is increasingly prevalent in industrial nations [10]. LSS can substantially impair i.a. patients' daily living, social participation, and physical function [11, 12]. Therapeutic approaches include pain reduction ranging from exercise- and physiotherapy to the short-term administration of analgesics and muscle relaxants [13, 14]. If those approaches fail, surgical intervention is an option $[13,14]$.

Many LSS patients suffer from considerable functional impairments that increase their risk of falling [7, 8] and subsequent adverse health events. Understanding the functional deficit of LSS patients may facilitate the planning of suitable therapy strategies. While studies of functional deficits explicitly in LSS patients $[4,5,7,15,16]$ are seldom, the balance performance of patients with LBP (presumably including LSS patients without specific nerve root irritations) has been more frequently assessed see [17]: several studies reported modified postural control in LBP patients compared to healthy control subjects [18-28]. Evaluating body excursion especially under dynamic and/or limited visual conditions revealed larger postural sway, represented by the root mean square (RMS) [17, 18, 21, 23, 29] and center of pressure (COP) displacement [19, 22, 25, 29]. Furthermore, the mean velocity (MV) of COP increases significantly in LBP patients when closing their eyes [17]. However, causes for LBP are manifold and may trigger different underlying mechanisms leading to balance impairments, such as pain, or afferent impairments. There is little knowledge about postural control behavior in the various subgroups of LBP patients.

Studies explicitly targeting LSS usually rely on COP evaluations during quiet stance $[4,5,7,15]$, while underlying sensorimotor mechanisms of stance regulation in LSS patients have been seldom assessed. Initial indications about altered body sway in LSS patients were published by Hanai et al. [15], who found that COP displacements after the onset of claudication increased and moved forward, in line with Sasaki et al. [7]. After surgery, patients' body sway decreased and approximated those of healthy subjects [15]. Other studies [4, 5, 7] supported the observation of abnormally large COP displacements in LSS patients, especially in anterior-posterior direction. Iversen et al. [4] found, in addition, significantly reduced functional-reach performance that may indicate impaired dynamic balance and an increased risk of falling. They suspect that LSS patients sense only greater body excursions in terms of a proprioceptive impairment, and need longer to switch between an assumed "open-loop"-control on the spinal level and an assumed "closed-loop" control on the cerebral level [4]. Both Iversen et al. [4] and Leinonen et al. [30] observed impaired lumbar movement perception that may result from a peripheral sensory loss. Furthermore, they propose an association between postural instability and abnormal motor-evoked potentials, while somatosensory conduction would not influence postural stability [30].

Based on those studies, we hypothesize that LSS patients reveal abnormal postural control that may be due to a reduced use of afferent, proprioceptive information. Instead, LSS patients may increase their dependence on space cues for postural stability. The present study was undertaken to i) assess the specific set of postural control deficits associated with LSS during spontaneous and externally perturbed stance and ii) to evaluate post-surgical changes in postural behavior. We applied an established postural control model [31-33] to extract relevant postural-control parameters that may reveal underlying sensorimotor mechanisms responsible for LSS patients' balance problems.

\section{Methods \\ Patients}

During 19 weeks, we consecutively enrolled 11 LSS patients and 15 healthy control subjects. Since our study follows a basic approach with the aim to understand fundamental postural control differences between LSS patients and healthy subjects, sample size could not be based on a previous power calculation. Inclusion criteria were: LSS diagnosis; hospitalized at Neurocenter - University of Freiburg, Germany and scheduled for surgical decompression. The lumbar spinal stenosis in our patients was due to an acquired degeneration and consecutive narrowing of the central canal leading to neurogenic claudication. We excluded patients suffering from radiculopathy. Other exclusion criteria were: additional disorders of the musculoskeletal system like lumbar disc herniation, synovial cyst, bone metastasis, lateral recess stenosis, recessusstenose, symptomatic cervical stenosis, paresis of knee extensors and hip flexors, or other sensorimotor impairments like polyneuropathy, eg due to diabetes mellitus, M. Parkinson, Myasthenia gravis, symptomatic stroke, clonus, cerebellar disorders, paraplegia, tumors or bleedings, peripheral vascular diseases like peripheral arterial occlusive disease; frailty, sarcopenia, $>80$ years. Therefore, all patients underwent detailed clinical neurological examination by an experienced neurologist. Control subjects were matched according to sex, age, weight and height. Table 1 summarizes our subjects' characteristic. 
Table 1 Patients' characteristic with $p$-values for differences

\begin{tabular}{|c|c|c|c|c|}
\hline & $\begin{array}{l}\text { Patients } \\
N=11\end{array}$ & $\begin{array}{l}\text { Patients post-surgery } \\
N=9\end{array}$ & $\begin{array}{l}\text { Control subjects } \\
N=15\end{array}$ & $P$ \\
\hline Age mean $\pm S D$ & $69 \pm 8$ & & $70 \pm 6$ & 0.89 \\
\hline $\operatorname{Sex}(m: f) N$ & $7: 4$ & & 8: 12 & 0.71 \\
\hline Weight $(\mathrm{kg})$ mean \pm SD & $84.3 \pm 17.9$ & & $77.9 \pm 14.1$ & 0.08 \\
\hline Height $(\mathrm{m})$ mean \pm SD & $1.71 \pm 0.7$ & & $1.69 \pm 1.0$ & 0.22 \\
\hline $\mathrm{BMI}\left(\mathrm{kg} / \mathrm{m}^{2}\right)$ mean $\pm \mathrm{SD}$ & $28.9 \pm 5.3$ & & $27.2 \pm 3.6$ & 0.14 \\
\hline \multicolumn{5}{|l|}{ Location of LSS N } \\
\hline$-L 2 / 3$ and $L 3 / 4$ & 1 & & & \\
\hline$-L 2 / 3, L 3 / 4$ and $L 4 / 5$ & 2 & & & \\
\hline$-\llcorner 3 / 4$ & 3 & & & \\
\hline$-L 3 / 4$ and $L 4 / 5$ & 2 & & & \\
\hline$-L 3 / 4, L 4 / 5$ and $L 5 / S 1$ & 1 & & & \\
\hline$-\llcorner 4 / 5$ & 2 & & & \\
\hline Time between symptom onset and surgery (years) mean \pm SD & $2.5 \pm 1.6$ & & & \\
\hline Time between diagnosis and surgery (months) mean \pm SD & $6.1 \pm 3.9$ & & & \\
\hline \multicolumn{5}{|l|}{ Symptoms $N$} \\
\hline - Claudicatio spinalis & 11 & & & \\
\hline - Reduced walking distance & 11 & & & \\
\hline - Subjective leg muscle weakness & 11 & & & \\
\hline - Inflected trunk position during walking & 11 & & & \\
\hline - Pain reduction induced by trunk flection and sitting position & 11 & & & \\
\hline \multicolumn{5}{|l|}{ Surgery $N$} \\
\hline - Minimally invasive osteoligamentous decompression & 7 & & & \\
\hline - Partially hemilaminectomy with additional osseous decompression & 4 & & & \\
\hline Days between pre- and post-assessment mean \pm SD & & $59 \pm 13$ & & \\
\hline Physiotherapy between surgery and post-assessment $N$ & & 9 & & \\
\hline
\end{tabular}

$P$ p-value, $S D$ standard deviation, $B M I$ body mass index, $L S S$ lumbar spinal stenosis

Patient-reported outcomes and postural control assessments were assessed pre-surgery (pre) and six to twelve weeks post-surgery (post) at routinely post-surgery check-up. During this interval, 9 patients received routine physiotherapy, with a focus on balance training as a standard measure for LSS patients after surgical decompression. All patient-related assessments were conducted by the same examiner following standardized measures.

This study was approved by the Ethics Commission of University Medical Center Freiburg. All subjects provided written informed consent to the experimental procedure in accordance with the Declaration of Helsinki.

\section{Functional tests and patient-reported outcomes (PROs)}

We clinically tested balance and mobility via the Performance Oriented Mobility Assessment (POMA) [34], Berg-Balance Scale (BBS) [35], the Clinical Test for Sensory Interaction in Balance (CTSIB) [36], the Functional Reach- [37], the Timed Up and Go (TUG)-, Chair Rising
(CRT)- and $10 \mathrm{~m}$ walking test [38] as well as spine mobility by determining the distance between the fingertips and ground.

Moreover, patients were asked to describe their pain intensity during resting and stress via visual analogue scale, and their maximum walking distance until claudicatio spinalis. Health-related quality of life was assessed via the SF-36 questionnaire (the short form (36) health survey) [39] and self-reported functional impairments via the Hannover questionnaire for functional disability caused by back pain (FFb-H-R) [40].

\section{Procedure}

Postural control assessments, ie spontaneous sway and perturbed stance were measured with a custom-built motion platform under two visual conditions, with eyes open and with eyes closed [41, 42]. Each trial lasted one minute. The participants were told to stand upright on the platform in comfortable shoes. Stance width was predetermined within a marked area. For safety reasons, 
participants had to hold two ropes hanging from the ceiling in crossed-arms position so that they could not perceive a somatosensory spatial orientation signal (for a more detailed setup description, see 32, [43]).

Spontaneous sway was measured on the non-moving platform. The COP sway path was detected with a force-transducing platform (Kistler platform type 9286, Winterthur, Switzerland).

Perturbed stance was measured on a moving platform to differentiate sensory contributions in reaction to external disturbances. We analyzed rotational tilts in the sagittal plane with the tilt axis passing through the participant's ankle joints. As the stimulus profile followed a pseudorandom stimuli (PRTS, pseudorandom ternary sequence [31]) with two peak angular displacements (stimulus amplitude: $0.5^{\circ}$ and $1^{\circ}$ peak-to-peak) and analyzed at eleven stimulus frequencies $(0.05,0.15,0.3,0.4$, $0.55,0.7,0.9,1.1,1.35,1.75$ and $2.2 \mathrm{~Hz}$ ).

Angular and translational excursions of the lower (hip-ankle: hip movement) and upper (shoulder-hip: shoulder movement) body segments and of the platform in space were measured using an optoelectronic motion-measuring device (Optotrak 3020, Waterloo, Canada) with markers attached to shoulder, hip, and the platform. Each marker consisted of three light-emitting diodes fixed to a rigid triangle. The triangles were fixed on the participant's hips and shoulders and to a rigid bar on the platform. 3-D angular and translational positions of the triangles were used to calculate marker positions. Optotrak $^{\circ}$ and Kistler output signals as well as the stimulus signals were transferred on-line to a computer system (IBM compatible Pentium ${ }^{\circ}$ ) via an analogue-digital converter at a sampling rate of $100 \mathrm{~Hz}$. We recorded all data with software programmed in LabView ${ }^{\circ}$ (National Instruments, Austin, Texas, USA).

\section{Model simulations}

The model simulations were implemented in Simulink/ MATLAB ${ }^{\mathrm{m}}$, as was an optimization procedure we used to identify model parameters [31, 33, 43]. In brief, the procedure varied defined model parameters, using the Matlab Optimization toolbox function "fminsearch" (which is based on the simplex search method of Nelder-Mead) in order to minimize the deviation between the simulated responses and corresponding experimental data. With each search's iteration, simulated responses to the pseudorandom stimulus cycles were obtained, the simulated data were analyzed in the same manner as the experimental data, and a scalar error function was evaluated representing the difference between simulated and experimental results. Then, the search procedure changed the parameters and the error function was re-evaluated. This sequence was repeated until parameters were identified yielding a minimum of the error function.

The model exploited here includes a negative feedback loop that relates body excursion detected by visual, vestibular, and proprioceptive sensors to a corrective torque via a neural controller (see [43]). We estimated the neural controller's parameters with proportional $(K p)$, derivative $(K d)$ and integral $(K i)$ contributions (PDI-controller). Neural controller gains are, in part, determined by mass and height of each subject's center of mass (COM) (see [31]).

Moreover, we derived time delay $(T d)$, proprioceptive sensory weight $(W p)$, and biomechanical elasticity (Ppas) and damping (Dpas) of the muscles and tendons. We fitted model simulations to experimental transfer functions under different stimulus amplitudes and visual conditions.

\section{Data analysis}

Data was analyzed off-line with custom-made software programmed in $\mathrm{MATLAB}^{\circ}$ (The MathWorks Inc., Natick, MA, USA). From the COP, lower (hip) and upper (shoulder) body excursions over time in anterior-posterior and medio-lateral sway directions, we calculated $R M S$ around the mean COP position. After differentiating the time series, we calculated $M V$. In addition, center frequency (CF) was extracted from the power spectrum (see also [44, 45]). Transfer functions from stimulus-response data were calculated via a discrete Fourier transform. Fourier coefficients of stimulus and response time series are used to determine GAIN and PHASE of the responses with respect to stimulus frequencies. We used these functions as the experimental data basis for model simulations using an upright-stance model. Further analysis was done using Microsoft Excel and statistic programs (JMP ${ }^{\circ}$, SAS Institute Inc., Cary, NC, USA). Statistical significance was tested by multivariate analysis of variance. The level of statistical significance was set at $p=0.05$. Relationships between the functional tests, RPOs and our postural control parameters obtained from platform measures were analyzed via the Pearson correlation test.

\section{Results}

\section{Spontaneous sway}

Our patients' postural sway was greater than our control subjects' (RMS: $\mathrm{F}=34.91 ; \mathrm{p}=<0.0001$; see Table 2 ). Post-surgery, RMS was significantly reduced $(\mathrm{F}=4.89 ; p$ $=0.0289$; see Table 2). This finding was mainly based on the eyes-open condition (interaction $\mathrm{F}=9.32 ; p=0.0028$ ) and in medio-lateral direction (interaction $\mathrm{F}=5.27 ; p=$ 0.0234, Fig. 1a).

Patients exhibited a higher MV at all conditions and qualities than control subjects $(\mathrm{F}=9.82 ; p=0.0019$; see Table 2). Group designation significantly interacted with visual condition $(\mathrm{F}=8.28 ; \mathrm{p}=<0.0042)$ and sway 
Table 2 Posturography

\begin{tabular}{|c|c|c|c|c|}
\hline & $\begin{array}{l}\text { Patients } \\
N=11\end{array}$ & $\begin{array}{l}\text { Patients post-surgery } \\
N=9\end{array}$ & $\begin{array}{l}\text { Control subjects } \\
N=15\end{array}$ & $P$ \\
\hline & Mean (SE) & $\Delta(\%)$ & Mean (SE) & \\
\hline \multicolumn{5}{|l|}{ Spontaneous sway } \\
\hline \multirow[t]{2}{*}{ RMS (cm) } & $0.56(0.02)$ & & $0.43(0.01)$ & $<0.000^{*}$ \\
\hline & & -5.3 & & $0.029^{*}$ \\
\hline \multirow[t]{2}{*}{$\mathrm{MV}(\mathrm{cm} / \mathrm{sec})$} & $0.72(0.04)$ & & $0.52(0.03)$ & $0.002^{*}$ \\
\hline & & -6.4 & & 0.244 \\
\hline \multirow[t]{2}{*}{$\mathrm{CF}(\mathrm{Hz})$} & $0.50(0.50)$ & & $0.44(0.01)$ & $0.001^{*}$ \\
\hline & & 4.6 & & 0.071 \\
\hline \multicolumn{5}{|l|}{ Perturbed stance } \\
\hline \multirow[t]{2}{*}{ Mean GAIN } & $1.85(0.02)$ & & $1.93(0.02)$ & $0.015^{*}$ \\
\hline & & 1.2 & & 0.482 \\
\hline \multirow[t]{2}{*}{ Mean PHASE $\left({ }^{\circ}\right)$} & $-98.83(2.22)$ & & $-119.09(1.90)$ & $<0.000^{*}$ \\
\hline & & 15.3 & & $<0.000^{*}$ \\
\hline \multicolumn{5}{|l|}{ Model parameters } \\
\hline \multirow[t]{2}{*}{$\mathrm{Ki}\left(\mathrm{s}^{-1} \cdot \mathrm{rad}^{-1}\right)$} & $74.95(2.63)$ & & $74.85(2.25)$ & 0.979 \\
\hline & & 5.2 & & 0.407 \\
\hline \multirow[t]{2}{*}{$\mathrm{Kp}\left(\mathrm{rad}^{-1}\right)$} & 1093.64 (39.11) & & 1047.63 (33.49) & 0.374 \\
\hline & & 0.0 & & 0.994 \\
\hline \multirow[t]{2}{*}{$\mathrm{Kd}\left(\mathrm{s} \cdot \mathrm{rad}^{-1}\right)$} & $307.39(8.61)$ & & $285.62(7.37)$ & 0.058 \\
\hline & & -4.3 & & 0.206 \\
\hline \multirow[t]{2}{*}{ Wp (\%) } & $61.04(2.59)$ & & $68.81(2.22)$ & $0.025^{*}$ \\
\hline & & 3.1 & & 0.595 \\
\hline \multirow[t]{2}{*}{$\mathrm{Td}$ (msec) } & $172(6.1)$ & & $172(5.3)$ & 0.919 \\
\hline & & -5.0 & & 0.344 \\
\hline \multirow[t]{2}{*}{ Ppas $\left(\operatorname{rad}^{-1}\right)$} & $82.25(3.37)$ & & $82.88(2.88)$ & 0.888 \\
\hline & & -9.0 & & 0.490 \\
\hline \multirow[t]{2}{*}{ Dpas $\left(s \cdot \operatorname{rad}^{-1}\right)$} & $55.93(1.42)$ & & $57.09(1.21)$ & 0.533 \\
\hline & & 6.3 & & 0.296 \\
\hline
\end{tabular}

*indicates a significant difference $p<0.05$

$\triangle$ post-surgery minus pre-surgery, $S E$ standard error, $R M S$ root means square, $M V$ mean velocity, $C F$ center frequency, $K i$ integral gain of the Neural Controller, $K p$ proportional gain (stiffness factor), $K d$ derivative gain (damping factor), Wp proprioceptive sensory weight, $T d$ feedback time delay, $P p a s$ passive stiffness factor, Dpas passive damping factor

direction $(\mathrm{F}=7.84 ; \mathrm{p}=<0.0054)$. Differences between patients and control subjects were especially pronounced with eyes closed and in the anterior-posterior direction (Fig. 1b). Post-surgery, we detected no effect on MV.

Patients swayed at higher frequencies than healthy subjects (CF: $\mathrm{F}=10.58 ; p=0.0013$; see Table 2 ), descriptively due to differences in anterior-posterior direction and with eyes closed (no significant interaction; see Fig. 1c). Post-surgery, we observed no significant changes in CF.

\section{Perturbed stance}

The transfer function between platform tilt and body angular displacement is characterized by GAIN and PHASE behavior.
Patients' GAIN was significantly smaller than that of control subjects $(\mathrm{F}=5.97 ; p<0.0146$; see Table 2$)$. In addition, group designation interacted significantly with frequency $(\mathrm{F}=3.53 ; p=0.0001)$ : at very low and high frequencies, patients showed a slightly larger GAIN than control subjects, while at lower and mid-range frequencies patients' GAIN was smaller than control subjects' (Fig. $2 \mathrm{~A}_{\mathrm{I}}-\mathrm{C}_{\mathrm{I}}$ ). We observed no interaction between GAIN and visual condition or rotational amplitude or body segment. Post-surgery, we observed no changes in GAIN behavior.

Patients displayed less PHASE lag than control subjects $(\mathrm{F}=48.29 ; p<0.0001$; see Table 2$)$. Moreover, group designation interacted significantly with rotational 


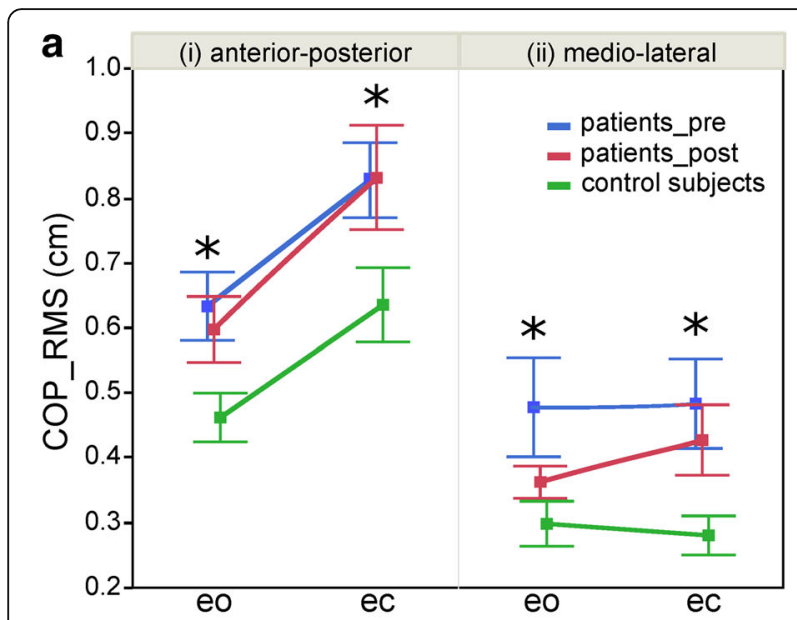

b

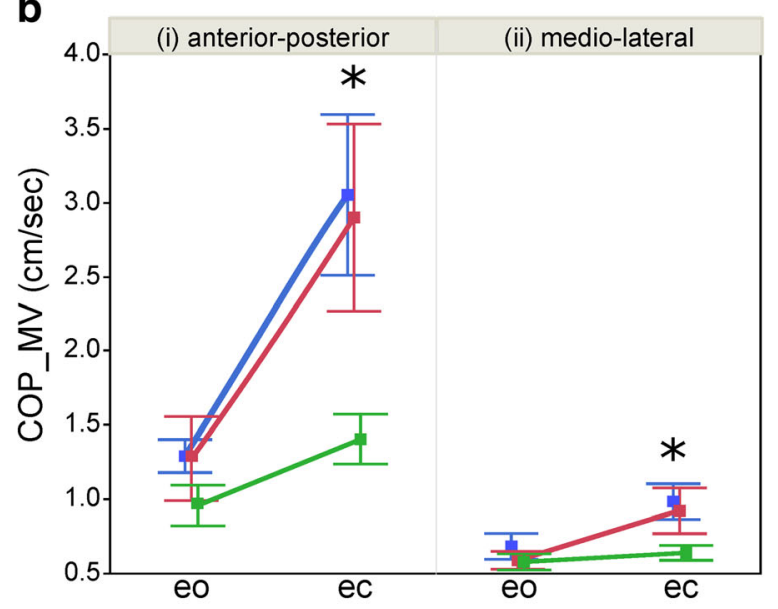

C

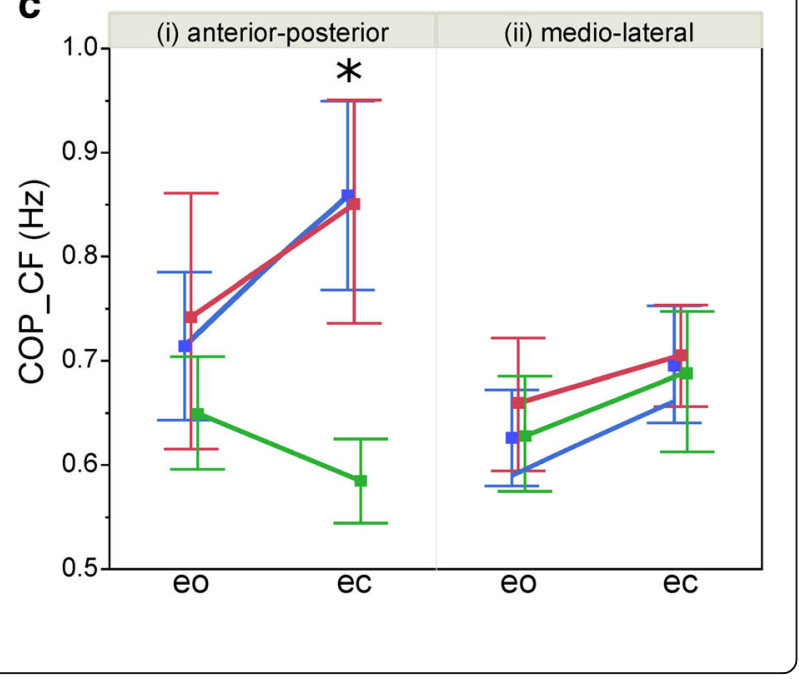

Fig. 1 Spontaneous sway. Center of pressure (COP) parameters: mean and standard error of (a) root mean square (RMS), (b) mean velocity (MV) and (c) center frequency (CF) of COP sway in (i) anterior-posterior and (ii) medio-lateral direction each for eyes open (eo) and eyes closed (ec) condition in healthy control subjects, patients before (pre) and after (post) surgery. Significant differences between healthy control subjects and patients before (pre) surgery are indicated with an asterisk

amplitude $\left(\mathrm{F}=45.32 ; \mathrm{p}=<0.0001 ;\right.$ Fig. $\left.2 \mathrm{~B}_{\mathrm{II}}\right)$. In particular, patients' PHASE behavior varied between the amplitudes meaning a smaller PHASE lag at $0.5^{\circ}$ compared to $1^{\circ}$, while control subjects acted similarly across amplitudes leading to a group difference at $0.5^{\circ}$ amplitude (Fig. $2 \mathrm{~B}_{\mathrm{II}}$ ). Patients demonstrated a greater PHASE lag post-surgery than pre-surgery $(\mathrm{F}=14.12 ; p=0.0002$; see Table 2). Patients' post-surgery PHASE behavior interacted with rotational amplitude $(\mathrm{F}=17.7 ; \mathrm{p}<0.0001)$ : PHASE advance was more pronounced at $0.5^{\circ}$ than at $1^{\circ}$; thus patients' post-surgery PHASE behavior approximated our control subjects' values at lower and mid-range frequencies, while the patients' (pre- and post-surgery) and control subjects' PHASE behavior at $1^{\circ}$ were nearly identical (Fig. $2 \mathrm{~B}_{\mathrm{II}}$ ). Furthermore, patients' post-surgery PHASE behavior interacted with body segment $(\mathrm{F}=5.5 ; \quad p=0.0042)$ : patients' PHASE lag post-surgery at hip increased at lower and mid-range frequencies approximating the range of control subjects (Fig. 2C $\mathrm{C}_{\mathrm{II}}$ ).

The following results are derived from the model-based approach described above, and present the relevant parameter differences between patients and control subjects.

We observed no significant effects for the integral (Ki), proportional $(\mathrm{Kp})$, and derivative $(\mathrm{Kd})$ parts of the neural controller (Table 2). However, the proportional (Kp) and derivative $(\mathrm{Kd})$ part were slightly larger in patients than in control subjects, whereas the $\mathrm{Kd}$ difference almost reached the significance level (0.058).

Time delay between stimulus and response (Td), passive muscle stiffness and damping (Ppas and Dpas) did not differ significantly between patients and control subjects (Table 2).

Only, the sensory weighting factor $\mathrm{Wp}$, indicating the proportion of proprioceptive vs. vestibular and visual cues, differed significantly between patients and control subjects $(\mathrm{F}=5.21 ; p=0.0246 ;$ Fig. 3). Whereas patients rely on average $61 \%$ on proprioceptive cues and hence $39 \%$ on spatial cues, control subjects rely $69 \%$ on proprioceptive and $31 \%$ on spatial cues (see Table 2). This Wp effect did not interact with visual condition or rotational amplitude, and was un affected by surgery, as with any other model parameters. 

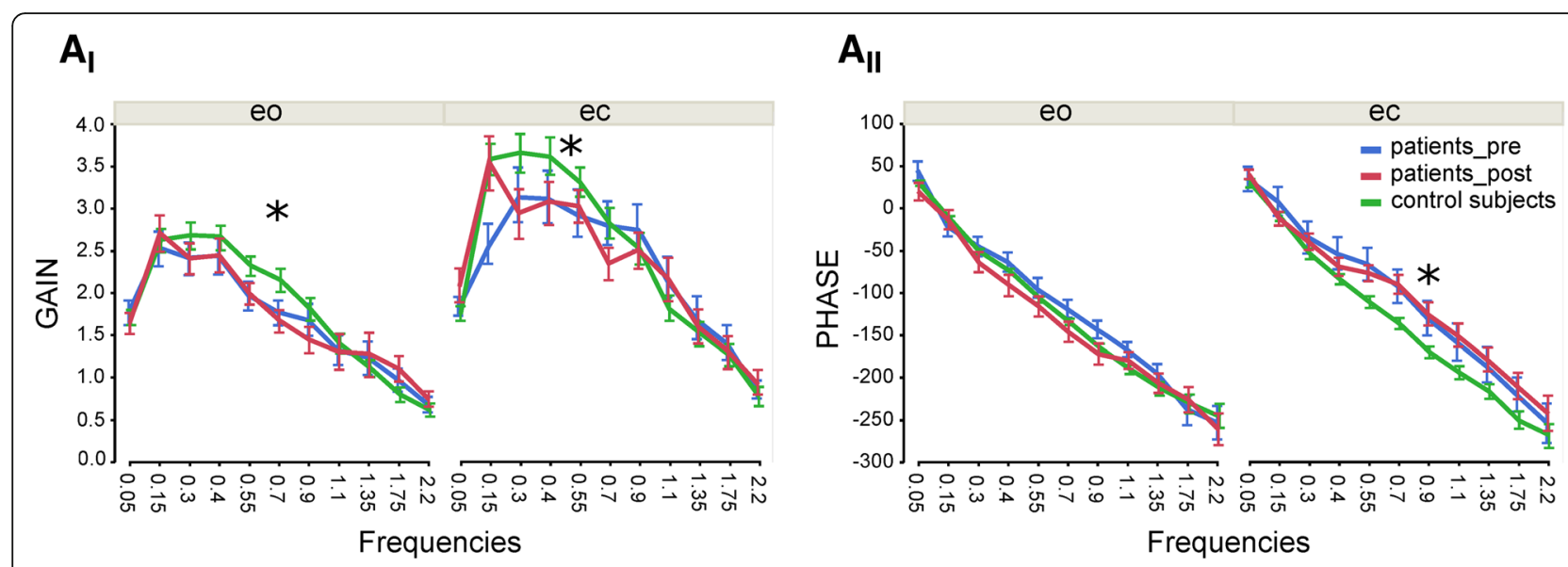

\section{$B_{1}$}

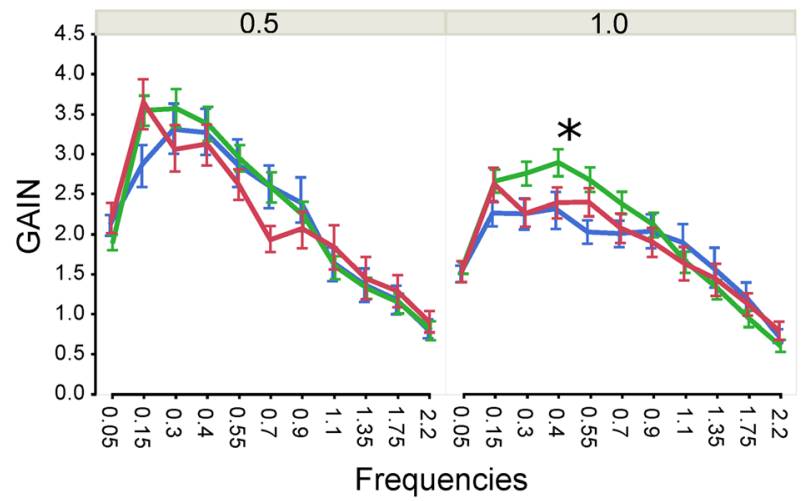

$B_{\text {II }}$

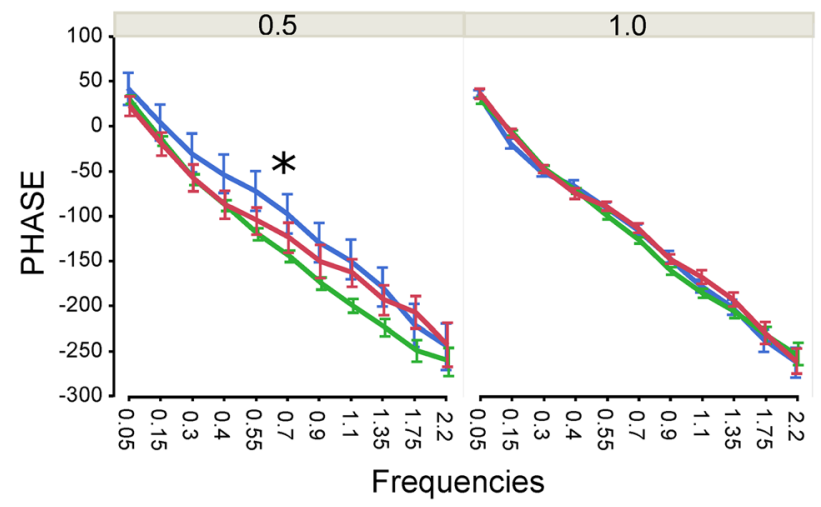

$C_{1}$

$\mathrm{C}_{\text {II }}$
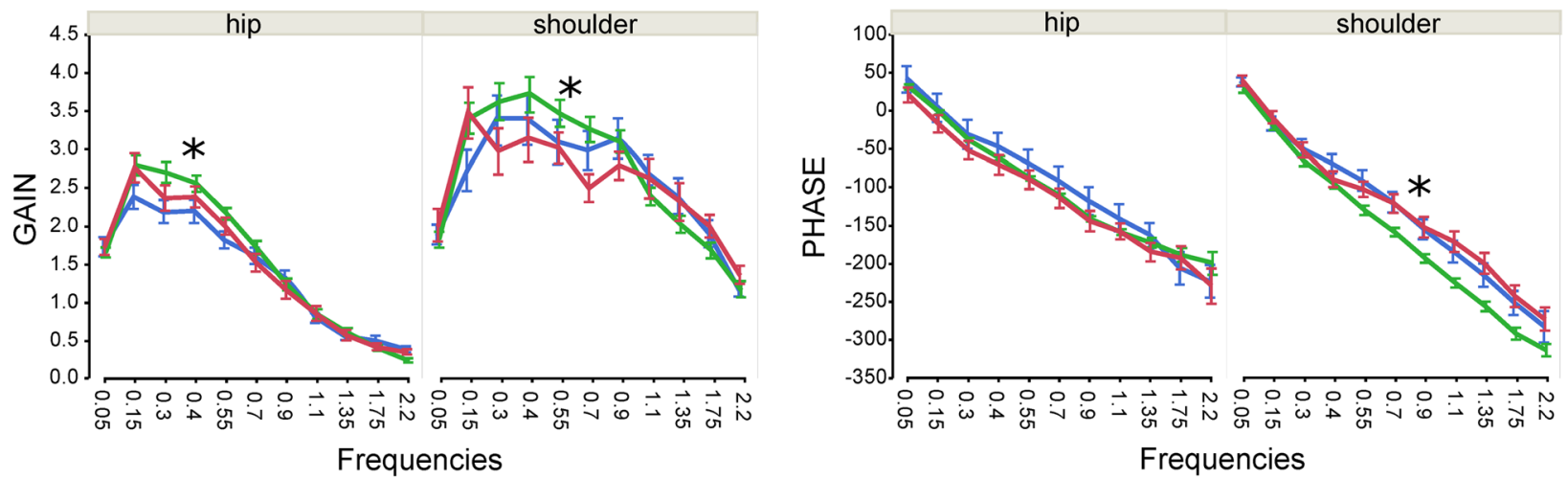

Fig. 2 Transfer functions. Mean and standard error of GAIN $\left(\mathbf{A}_{\mid}-\mathbf{C}_{\mid}\right)$and PHASE $\left(\mathbf{A}_{\|}-\mathbf{C}_{\|}\right)$behavior as a function of frequency for eyes-open (eo) and eyes-closed (ec) condition (A), for $0.5^{\circ}$ and $1^{\circ}$ rotational amplitude $(\mathbf{B})$ and body segments $(\mathbf{C})$ in healthy control subjects, patients before (pre) and after (post) surgery. Significant differences between healthy control subjects and patients before (pre) surgery are indicated with an asterisk

\section{Functional tests and PROs}

Patients' pre-surgical functional status was not considerably impaired (Table 3). After surgery, we detected no significant change in functional tests. However, among PROs, patients reported impaired function (FFb-H-R), reduced quality of life (SF-36), limited maximum walking distance and high pain intensity during stress (Table $3)$. After surgery, pain intensity during rest ( $\mathrm{F}=5.73$; $p=$ $0.0436)$ and stress $(\mathrm{F}=43.75 ; p=0.0002)$ was significantly reduced; patients reported a considerably longer 


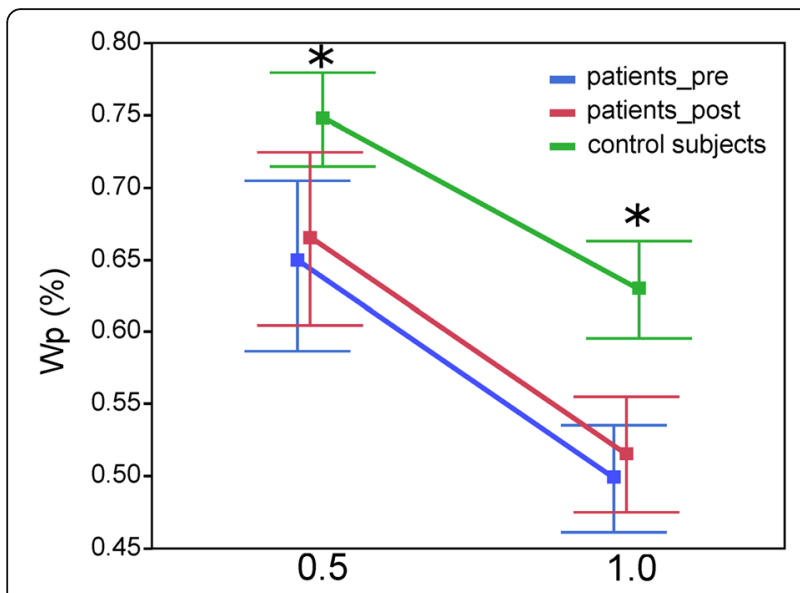

Fig. 3 Model parameters. Mean and standard error of the proprioceptive sensory weight (Wp) for $0.5^{\circ}$ and $1^{\circ}$ rotational amplitude in healthy control subjects, patients before (pre) and after (post) surgery. Significant differences between healthy control subjects and patients before (pre) surgery are indicated with an asterisk

walking distance $(\mathrm{F}=7.44 ; p=0.0295)$ and improved function $(\mathrm{F}=8.76 ; p=0.0182)$, while quality of life was unaffected.

\section{Correlations}

Our correlation analysis revealed a strong interrelation between the functional tests BBS, $10 \mathrm{~m}$ walking test, POMA and TUG ( $\mathrm{r}>0.7, p<0.001)$, as well as between CTSIB and POMA $(\mathrm{r}=0.59, p<0.01)$, while CRT correlated only with the $10 \mathrm{~m}$ walking test $(\mathrm{r}=0.54, \mathrm{p}=0.02)$ and TUG $(\mathrm{r}=0.65, \mathrm{p}<0.01)$. In contrast, functional reach and spine mobility did not correlate with other functional tests or with PROs or with experimental results. Based on the largely strong correlations between BBS, $10 \mathrm{~m}$ walking test, POMA, TUG, CRT, and CTSIB we used a principal component (PC) analysis to condense these functional tests (FT) into one variable (PC-FT).

Concerning PROs, we noted significant correlations between FFb-H-R, which represents the center of PROs' correlations and reflects many different aspects of patient-reported functional impairments, and pain intensity during stress as well as max. Walking

Table 3 Functional tests and patient-reported outcomes (PROs)

\begin{tabular}{|c|c|c|c|c|}
\hline & $\begin{array}{l}\text { Patients } \\
N=9\end{array}$ & $\begin{array}{l}\text { Patients post-surgery } \\
N=9\end{array}$ & $\begin{array}{l}\text { Mean difference } \\
(95 \% \mathrm{Cl})\end{array}$ & P \\
\hline & Mean & \multicolumn{3}{|l|}{ Mean } \\
\hline \multicolumn{5}{|l|}{ Functional tests } \\
\hline Spine mobility $(\mathrm{cm})^{a}$ & 9.89 & 10.00 & $0.11(4.13--3.91)$ & 0.951 \\
\hline Functional reach $(\mathrm{cm})^{\mathrm{b}}$ & 32.05 & 34.11 & $2.06(11.95--7.84)$ & 0.645 \\
\hline BBS (scale max. 56) ${ }^{c}$ & 52.67 & 54.33 & $1.67(4.27--0.94)$ & 0.179 \\
\hline $10 \mathrm{~m}$ walking test (sec) & 6.8 & 5.7 & $-1.1(0.3--2.4)$ & 0.099 \\
\hline CRT $(\mathrm{sec})^{\mathrm{d}}$ & 16 & 18 & $2(5--1)$ & 0.250 \\
\hline CTSIB (scale 6-24) & 9.3 & 9.6 & $0.2(1.4--0.9)$ & 0.665 \\
\hline POMA (scale max. 28) ${ }^{f}$ & 26.7 & 27.3 & $0.67(1.5--0.2)$ & 0.111 \\
\hline TUG $(\mathrm{sec})^{\mathrm{g}}$ & 10.0 & 8.9 & $-1.1(0.5--2.7)$ & 0.154 \\
\hline \multicolumn{5}{|l|}{ PROs } \\
\hline Pain intensity during rest (VAS scale $0-10$ ) & 2.11 & 0.67 & $-1.44(-0.05--2.84)$ & $0.044^{*}$ \\
\hline Pain intensity during stress (VAS scale $0-10$ ) & 7.56 & 3.67 & $-3.89(-2.53--5.35)$ & $<0.000^{*}$ \\
\hline Max. walking distance (m) & 329 & 4775 & $4446(8302-591)$ & $0.030^{*}$ \\
\hline FFb-H-R (score max. 100\%) ${ }^{h}$ & 60.7 & 73.2 & $-12.5(-2.8--22.2)$ & $0.018^{*}$ \\
\hline SF-36 (score 0-100\%) $)^{i}$ & 49.3 & 56.4 & $7.2(23.0--8.6)$ & 0.326 \\
\hline \multicolumn{5}{|c|}{ 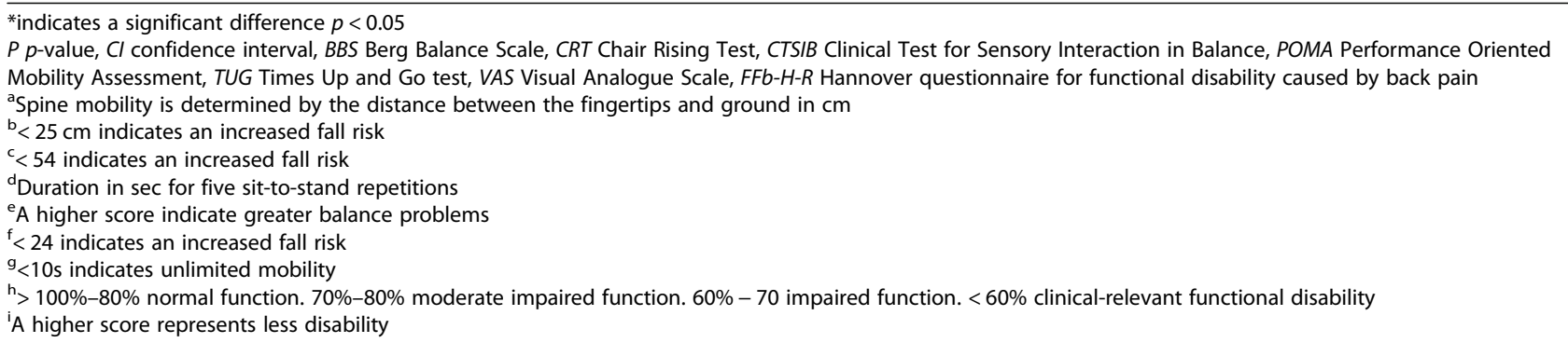 } \\
\hline
\end{tabular}


distance $(\mathrm{r}>0.5, p<0.05)$. Moreover, FFb-H-R correlated strongly with SF-36 $(\mathrm{r}=0.63, p=0.005)$. Walking distance correlated strongly with pain intensity during stress $(\mathrm{r}=0.79, \mathrm{p}<0.001)$ and with SF-36 $(\mathrm{r}=$ 0.49, $p=0.05$ ).

Pain intensity during rest showed no interrelation to other PROs, any functional tests, or experimental results.

Furthermore, we observed a strong correlation between experimental results, ie PHASE and GAIN ( $\mathrm{r}=$ $0.59, p=0.009)$, while the model parameter representing proprioception, i.e. Wp, correlated only with GAIN ( $\mathrm{r}=$ $0.71, p=0.001)$. GAIN and PC-FT also correlated strongly $(\mathrm{r}=0.62, p=0.006)$, as did GAIN and FFb-H-R $(p=0.52, \quad p=0.027)$. FFb-H-R correlated also with PHASE $(\mathrm{r}=0.50, p=0.035)$ and with PC-FT $(\mathrm{r}=0.67, p$ $=0.003)$.

Spontaneous sway results did not correlate with perturbed stance results, with functional tests, or with PROs.

\section{Discussion}

Functional impairments are common among LSS patients $[3,6,9]$. Some authors have suggested [4, 30] that these impairments may be related to peripheral sensory loss. However, it remains unclear which postural mechanisms are directly involved. Possible candidates are impaired motor actuator, sensorimotor time delay, impaired weighting of visual, vestibular and proprioceptive input, passive muscle elasticity, and damping. This ambiguity motivated us to conduct the present study. Our study's aim was to identify the postural control parameters that may reveal underlying sensorimotor mechanisms responsible for LSS patients' balance problems. Moreover, we aimed to monitor pre-post-surgical changes in these parameters. We hypothesized that the main effect in LSS patients concerns the altered weighting of sensory information. In particular, we expected an altered use of proprioceptive information in LSS patients as compared to healthy control subjects.

In line with earlier studies reporting postural instability in patients suffering from LSS $[4,5,15]$, we detected altered postural behavior in LSS patients. Interestingly, we were able to relate these findings to a modified use of sensory information and to timing aspects of postural reactions. Surgery and post-surgery rehabilitation mainly affected these timing aspects of postural behavior. In addition, the clinical outcome of LSS patients post-surgery was improved, reflected by reduced patient-reported functional impairments.

Since this study serve as basis for further investigations aiming to understand the underlying sensorimotor mechanisms responsible for LLS patients' postural instability, sample size was not based on a previous power calculation. The small sample size may limit our study's results regarding stronger differences between groups. Furthermore, we propose to monitor post-surgery physiotherapy measures or to intervene specifically in order to control influencing factors on postural stability, and to expand the observation duration to detect long-term effects.

\section{Spontaneous sway}

Spontaneous sway measurements revealed that LSS patients' postural sway was greater, faster and at higher frequencies compared to matched healthy control subjects. Those alterations have been also described in LBP patients [18-29] and elsewhere in LSS patients [4, 5]. Abnormal spontaneous sway was associated with a higher risk of falling in LSS patients [7]. Our PROs confirmed functional limitations in LSS that considerably affect patients' quality of life, and additionally indicate stress-dependent worsening of impairments.

Post-surgery, postural sway (as measured by RMS) was reduced and approximated the values of control subjects which paralleled findings from an earlier study [15]. This reduction may indicate that patients achieved better postural stability post-surgery following the decompression of lumbar radices, which carry the motor and sensory information for lower extremity control. Specifically, the RMS reduction after surgery and rehabilitation measures may be a consequence of improved peripheral proprioception. Since surgery significantly reduced pain intensity and patient-reported functional limitations, as expected, this also may contribute to improved stance stability, which is also influenced by rehabilitation measures.

\section{Perturbed stance and model-based analysis}

In addition to investigating spontaneous sway, we assessed postural reactions to external stimuli. Specifically, we compared patients' postural sway to continuous pseudorandom platform tilts in anterior-posterior direction. These raw angular traces were transformed into transfer functions in the frequency domain, reflecting patients' reactive body excursions as a function of stance perturbation. These transfer functions revealed a smaller GAIN in patients than control subjects, especially in lower and mid-range frequencies. In other words, patients' postural reactions were, on average, smaller than control subjects'. Furthermore, patients presented a smaller PHASE lag, representing a relatively earlier postural reaction to external stimuli than control subjects. Interestingly, PHASE differences between patients and control subjects were amplitude-dependent (apparent at $0.5^{\circ}$, but vanished at $1^{\circ}$ ). Patients' PHASE lag at $0.5^{\circ}$ corresponded to that of control subjects at $1^{\circ}$, meaning that 
patients reacted to smaller platform excursions like control subjects to greater excursions. Post-surgery, patients approximated the PHASE behavior of control subjects, which is mainly based on changes at 0.5 rotational amplitude and at hip movement.

On a first glance, LSS patients' smaller and earlier reactive body excursions, as compared to control subjects, do not seem to be disadvantageous for postural stability. Even more surprising, post-surgery we observed a prolongation of postural reactions in LSS patients, represented by an increased PHASE lag. How could this prolongation be related to the fact that patients achieved enhanced posture stability post-surgery, as our spontaneous sway findings demonstrate (RMS)?

The transfer function findings reported above formed a basis for a model-based data interpretation: Extracting model-parameters after fitting simulated to experimental transfer functions revealed slightly more pronounced velocity control (damping) in the postural system (Kd) in patients compared to control subjects. Kd results are in line with the patients' aforementioned PHASE behavior. Furthermore, patients use significantly less proprioceptive information, as reflected by smaller values for proprioceptive weight (Wp), than control subjects. No other model parameters differed between groups.

Following the assumption that LSS patients weigh their sensory information differently than healthy subjects, we hypothesized that patients rely relatively more on space cues and less on proprioceptive cues, as they suffer from stenosis-induced afferent dysfunction. Our GAIN results support this assumption, since our patients revealed a smaller GAIN, indicating their stronger orientation towards space coordinates and less dependency on platform movements [31, 43]. In contrast, healthy subjects integrated more proprioceptive information for stance regulation resulting in a greater GAIN. These findings are emphasized by our model-based approach, where we found patients' proprioceptive cues (Wp) down-weighted and as a result, space cues implicitly up-weighted when compared to healthy subjects. However, up-weighting space cues implies a functional disadvantage, as the vestibular system carries a larger amount of sensory noise [46], leading to less accurate stance regulation. There is evidence that larger platform perturbations generally lead to a relative smaller GAIN, stronger orientation on space cues, a smaller PHASE lag, and greater velocity control (Kd) [47] to ensure stance stability. We assume that patients' postural behavior might represent a compensation strategy, where patients try to prevent excessive and rapid changes in postural position already at small excursions by reducing PHASE lag due to their stenosis-caused physiological limitations and related pain. Additionally, as mentioned earlier, afferent information might be inadequate, which could be a reason why patients down-weigh proprioception. Patients compensate for their afferent dysfunction by staying within a more stable and secure range of regulation [48], while accepting disadvantages in the accuracy of postural control through increased vestibular noise. Surgery alleviated physiological limitations responsible for this compensation, leading to a PHASE-shift towards healthy subjects' postural behavior.

The concentration of the intervention-induced PHASE effect on the hip joint may indicate, in addition, a change in hip-strategy $[49,50]$. Concerning LBP patients, some authors reported that these patients exhibit a less effective hip strategy than healthy subjects [24, 27, 51]. According to those findings, we speculate that patients stiffened their hip joint pre-surgery by enhancing muscular co-contractions due to the aforementioned worries about safety. Post-surgery, this stiffness may be relaxed to enable larger body excursions [50], as surgery directly alleviates their primary impairments, eg pain $[3,9,52]$.

Considering patients' afferent dysfunction before surgery, one would assume that motor latency as reaction of the perturbation would be prolonged. However, time delay (Td) extracted from the model-based approach did not differ between groups, highlighting the assumption that altered PHASE behavior as a function of time is not primarily caused by physiological impairments but rather by a compensation strategy, ie altered timing for stance regulation.

Additionally, the model-based total effort to correct the difference between actual and desired body angle (represented by the model parameter $\mathrm{Kp}$ ) was similar in both groups, indicating that the intensity of motor response was also not unaltered in LSS patients. We therefore assume that the stenosis-caused efferent dysfunction was less pronounced in our patients and did not lead to a fundamental deficit in relation to their stance regulation.

\section{Correlations}

When comparing our experimental results with clinical tests of motor function and patient-reported outcomes, one is tempted to associate the spontaneous sway abnormalities, ie larger (RMS) and faster (MV) postural sway to the other functional disadvantages pre-surgery. In the literature, these spontaneous sway abnormalities are reported to be associated with postural instability and functional impairments [4, 53]. Interestingly, our spontaneous sway parameters neither correlated with patient-reported outcomes, nor with functional tests, nor with measures derived from perturbed stance. In contrast, measures from perturbed stance significantly correlate with both patient-reported functional 
outcomes (FFb-H-R) and specific functional tests, which in turn correlated strongly. The significant correlation between $\mathrm{FFb}-\mathrm{H}-\mathrm{R}$ and many functional tests (PC-FT: POMA [34], BBS [35], CTSIB [36], TUG-, CRT- and 10 $\mathrm{m}$ walking test [38]) indicate that these functional tests mainly determine the perception of functional impairments in patients [54], while other tests, ie the Functional Reach test [37], or spine mobility (distance between the fingertips and ground) seem to reflect other functionality dimensions. We claim that the close correlation between our experimental results from perturbed stance, many functional tests (PC-FT), and the patient-reported deficits may be applied in future to lower the number of tests necessary to accurately assess all facets of postural impairments.

In general, our data lead us to conclude that patients' postural reactions following external perturbations are suitable to precisely quantify most of their postural impairments unlike spontaneous sway measures. However, taking spontaneous sway measurements is the current standard when assessing patients' functional status [18$25,29]$. Based on our results, we suspect that parameters recorded during quiet stance fail to capture patients' gait and stance disturbances sufficiently - thus we strongly propose applying external perturbation when assessing patients' balance ability.

\section{Conclusion}

In summary, LSS patients reported significant functional impairments that can be captured by our perturbed stance experiments. Analyzing perturbed stance revealed alterations in the timing of postural reactions and in the down-weighting of proprioceptive cues, possibly due to stenosis-caused afferent dysfunctions. In return, patients involuntarily accept functional disadvantages when up-weighting space cues. They may be compensating for this disadvantage by an earlier motor reaction as response to body excursions to prevent excessive and rapid changes in posture position for safety reasons. After surgery and few weeks rehabilitation, patients reported fewer impairments. Furthermore, these interventions corrected for the compensation mechanism, as patients approximated the postural behavior of healthy subjects in terms of timing aspects. However, sensory weighting was unaffected at follow-up. Thus, we propose that post-surgery rehabilitation in LSS patients should focus on interventions ameliorating the proprioceptive part of postural control, eg on balance exercises on unstable surfaces entailing reduced visual input.

\section{Abbreviations}

BBS: Berg-Balance Scale; CF: Center frequency; COM: Center of mass; COP: Center of pressure; CRT: Chair Rising Test; CTSIB: Clinical Test for Sensory Interaction in Balance; Dpas: Passive damping factor; FFb-HR: Hannover questionnaire for functional disability caused by back pain;
Kd: Derivative gain (damping factor); Ki: Integral gain of the Neural Controller; Kp: Proportional gain (stiffness factor); LBP: Low back pain; LSS: Lumbar spinal stenosis; MV: Mean velocity; PC-FT: Principal component (PC) of specific functional tests (FT); POMA: Performance Oriented Mobility Assessment; Ppas: Passive stiffness factor; PROs: Patient-reported outcomes; RMS: Root mean square; SF-36: Short form (36) health survey; Td: Feedback time delay; TUG: Timed Up and Go; Wp: Proprioceptive sensory weight

\section{Acknowledgements}

We thank the patients and control individuals for their collaboration. The article processing charge was funded by the German Research Foundation (DFG) and the Albert Ludwigs University Freiburg, Germany, in the funding program Open Access Publishing.

\section{Funding}

C.M. was partially funded by the European Union grants FP7 (EMBALANCE: Grant Agreement no 610454) and H2020-SC1-2017-CNECT-1 (HOLOBALANCE: Project ID 769574; HOLOgrams for personalised virtual coaching and motivation in an ageing population with BALANCE disorders). S.K., V.B., D.D. and C.M. were partially funded by the Brainlinks-Braintools Cluster of Excellence funded by the German Research foundation (DFG, grant no ADV139).

The funders neither played a role in the study design, nor in data collection, analysis and interpretation, the writing process of the manuscript or the decision to submit for publication.

\section{Availability of data and materials}

This article only includes summarized data from this study. Raw data is available from the corresponding author upon reasonable request.

\section{Authors' contributions}

SK and CM drafted the manuscript, analyzed and interpreted the data. CM and UH designed the study. VB recruited patients, collected and analyzed data. UH assisted patients' recruitment. DD supervised and assisted the measurements as well as data analysis. All authors made contributions to the paper. All authors read and approved the final manuscript.

\section{Ethics approval and consent to participate}

Ethics approval was granted by the Ethics Committee of the University of Freiburg (256/01). Each patient had to sign written informed consent prior to inclusion.

\section{Consent for publication}

Not applicable.

\section{Competing interests}

The authors have no competing interests to declare.

\section{Publisher's Note}

Springer Nature remains neutral with regard to jurisdictional claims in published maps and institutional affiliations.

\section{Author details}

${ }^{1}$ Department of Neurology, Faculty of Medicine and Medical Center University of Freiburg, Freiburg, Germany. ${ }^{2}$ Department of Neurosurgery, Faculty of Medicine and Medical Center - University of Freiburg, Freiburg, Germany.

Received: 23 October 2018 Accepted: 6 March 2019

Published online: 01 May 2019

References

1. Katz JN, Harris MB. Clinical practice. Lumbar spinal stenosis. N Engl J Med. 2008:358:818-25.

2. Kalichman L, Cole R, Kim DH, Li L, Suri P, Guermazi A, et al. Spinal stenosis prevalence and association with symptoms: the Framingham study. Spine J Off J North Am Spine Soc. 2009;9:545-50.

3. Iversen MD, Katz JN. Examination findings and self-reported walking capacity in patients with lumbar spinal stenosis. Phys Ther. 2001;81:1296-306. 
4. Iversen MD, Kale MK, Sullivan JT Jr. Pilot case control study of postural sway and balance performance in aging adults with degenerative lumbar spinal stenosis. J Geriatr Phys Ther 2001. 2009;32:15-21.

5. Truszczyńska A, Drzał-Grabiec J, Trzaskoma Z, Rapała K, Tarnowski A, Górniak K. A comparative analysis of static balance between patients with lumbar spinal canal stenosis and asymptomatic participants. J Manip Physiol Ther. 2014;37:696-701.

6. Thornes E, Robinson HS, Vøllestad NK. Degenerative lumbar spinal stenosis and physical functioning: an exploration of associations between selfreported measures and physical performance tests. Disabil Rehabil. 2018;40: 232-7.

7. Sasaki K, Senda M, Katayama Y, Ota H, Matsuyama Y. Characteristics of postural sway during quiet standing before and after the occurrence of neurogenic intermittent claudication in female patients with degenerative lumbar Spinal Canal stenosis. J Phys Ther Sci. 2013;25:675-8.

8. Kim H-J, Chun H-J, Han C-D, Moon S-H, Kang K-T, Kim H-S, et al. The risk assessment of a fall in patients with lumbar spinal stenosis. Spine. 2011;36:E588-92.

9. Lin S-I, Lin R-M. Disability and walking capacity in patients with lumbar spinal stenosis: association with sensorimotor function, balance, and functional performance. J Orthop Sports Phys Ther. 2005;35:220-6.

10. Manchikanti L, Singh V, Falco FJE, Benyamin RM, Hirsch JA. Epidemiology of low back pain in adults. Neuromodulation J Int Neuromodulation Soc. 2014; 17(Suppl 2):3-10.

11. AHRQ. Treatment of Degenerative Lumbar Spinal Stenosis. In: Summary, Evidence Report/Technology Assessment. Rockville: Agency for Healthcare Research and Quality (US); 2001.

12. Ammendolia C, Stuber KJ, Rok E, Rampersaud R, Kennedy CA, Pennick V, et al. Nonoperative treatment for lumbar spinal stenosis with neurogenic claudication. Cochrane Database Syst Rev. 2013.

13. Binder DK, Schmidt MH, Weinstein PR. Lumbar Spinal Stenosis. Semin Neurol. 2002;22:157-66.

14. Lee SY, Kim T-H, Oh JK, Lee SJ, Park MS. Lumbar stenosis: a recent update by review of literature. Asian Spine J. 2015;9:818-28.

15. Hanai K, Ishii K, Nojiri H. Sway of the center of gravity in patients with spinal canal stenosis. Spine. 1988;13:1303-7.

16. Thornes E, Robinson HS, Vøllestad NK. Dynamic balance in patients with degenerative lumbar spinal stenosis; a cross-sectional study. BMC Musculoskelet Disord. 2018;19:192.

17. Ruhe A, Fejer R, Walker B. Center of pressure excursion as a measure of balance performance in patients with non-specific low back pain compared to healthy controls: a systematic review of the literature. Eur Spine J. 2011; 20:358-68.

18. Mientjes MI, Frank JS. Balance in chronic low back pain patients compared to healthy people under various conditions in upright standing. Clin Biomech Bristol Avon. 1999:14:710-6.

19. Hamaoui A, Do MC, Bouisset S. Postural sway increase in low back pain subjects is not related to reduced spine range of motion. Neurosci Lett. 2004;357:135-8.

20. Hamaoui A, Do MC, Poupard L, Bouisset S. Does respiration perturb body balance more in chronic low back pain subjects than in healthy subjects? Clin Biomech Bristol Avon. 2002;17:548-50.

21. della Volpe R, Popa T, Ginanneschi F, Spidalieri R, Mazzocchio R, Rossi A. Changes in coordination of postural control during dynamic stance in chronic low back pain patients. Gait Posture. 2006;24:349-55.

22. Popa T, Bonifazi M, Della Volpe R, Rossi A, Mazzocchio R. Adaptive changes in postural strategy selection in chronic low back pain. Exp Brain Res. 2007; 177:411-8.

23. Brumagne $S$, Janssens L, Janssens E, Goddyn L. Altered postural control in anticipation of postural instability in persons with recurrent low back pain. Gait Posture. 2008;28:657-62

24. Brumagne S, Janssens L, Knapen S, Claeys K, Suuden-Johanson E. Persons with recurrent low back pain exhibit a rigid postural control strategy. Eur Spine J. 2008;17:1177-84.

25. Mann L, Kleinpaul JF, Pereira Moro AR, Mota CB, Carpes FP. Effect of low back pain on postural stability in younger women: influence of visual deprivation. J Bodyw Mov Ther. 2010;14:361-6.

26. Leitner C, Mair P, Paul B, Wick F, Mittermaier C, Sycha T, et al. Reliability of posturographic measurements in the assessment of impaired sensorimotor function in chronic low back pain. J Electromyogr Kinesiol. 2009;19:380-90.

27. Johanson E, Brumagne $\mathrm{S}$, Janssens L, Pijnenburg M, Claeys K, Pääsuke M. The effect of acute back muscle fatigue on postural control strategy in people with and without recurrent low back pain. Eur Spine J. 2011;20: 2152-9.

28. Willigenburg NW, Kingma I, van Dieën JH. Precision control of an upright trunk posture in low back pain patients. Clin Biomech. 2012; 27:866-71.

29. Lafond D, Champagne A, Descarreaux M, Dubois J-D, Prado JM, Duarte M. Postural control during prolonged standing in persons with chronic low back pain. Gait Posture. 2009;29:421-7.

30. Leinonen V, Määttä S, Taimela S, Herno A, Kankaanpää M, Partanen J, et al. Impaired lumbar movement perception in association with postural stability and motor- and somatosensory-evoked potentials in lumbar spinal stenosis. Spine. 2002;27:975-83.

31. Peterka RJ. Sensorimotor integration in human postural control. J Neurophysiol. 2002;88:1097-118.

32. van Kordelaar J, Pasma JH, Cenciarini M, Schouten AC, van der Kooij H, Maurer $C$. The reliance on vestibular information during standing balance control decreases with severity of vestibular dysfunction. Front Neurol. 2018;9:371.

33. Engelhart D, Pasma JH, Schouten AC, Meskers CGM, Maier AB, Mergner T, et al. Impaired standing balance in elderly: a new engineering method helps to unravel causes and effects. J Am Med Dir Assoc. 2014;15:227.e1-6.

34. Tinetti ME. Performance-oriented assessment of mobility problems in elderly patients. J Am Geriatr Soc. 1986;34:119-26.

35. Berg K, Wood-Dauphine S, Williams Jl, Gayton D. Measuring balance in the elderly: preliminary development of an instrument. Physiother Can. 1989: 304-11.

36. Shumway-Cook A, Horak FB. Assessing the influence of sensory interaction of balance. Suggestion from the field. Phys Ther. 1986; 66:1548-50.

37. Weiner DK, Duncan PW, Chandler J, Studenski SA. Functional reach: a marker of physical frailty. J Am Geriatr Soc. 1992;40:203-7.

38. Bennell K, Dobson F, Hinman R. Measures of physical performance assessments: self-paced walk test (SPWT), stair climb test (SCT), sixminute walk test (6MWT), chair stand test (CST), timed up \& go (TUG), sock test, lift and carry test (LCT), and Car task. Arthritis Care Res. 2011;63(Suppl 11):S350-70.

39. Bullinger $M$, Morfeld $M$, Kohlmann T, Nantke J, van den Bussche $H$, Dodt $B$, et al. SF-36 health survey in rehabilitation research. Findings from the north German network for rehabilitation research, NVRF, within the rehabilitation research funding program. Rehabil. 2003;42:218-25.

40. Kohlmann T, Raspe HH. Der Funktionsfragebogen Hannover zur alltagsnahen Diagnostik der Funktionsbeeinträchtigung durch Rückenschmerzen (FFbH-R) [Hannover questionnaire for functional disability caused by back pain]. Rehabilitation (Stuttg). 1996;35:I-VIII.

41. Maurer C, Mergner T, Peterka RJ. Multisensory control of human upright stance. Exp Brain Res. 2006;171:231-50.

42. Cnyrim C, Mergner T, Maurer C. Potential roles of force cues in human stance control. Exp Brain Res. 2009;194:419-33.

43. Wiesmeier IK, Dalin D, Wehrle A, Granacher U, Muehlbauer T, Dietterle J, et al. Balance training enhances vestibular function and reduces overactive proprioceptive feedback in elderly. Front Aging Neurosci. 2017:9:273.

44. Maurer C, Peterka RJ. A new interpretation of spontaneous sway measures based on a simple model of human postural control. J Neurophysiol. 2005; 93:189-200

45. Prieto TE, Myklebust JB, Hoffmann RG, Lovett EG, Myklebust BM. Measures of postural steadiness: differences between healthy young and elderly adults. IEEE Trans Biomed Eng. 1996;43:956-66.

46. van der Kooij H, Peterka RJ. Non-linear stimulus-response behavior of the human stance control system is predicted by optimization of a system with sensory and motor noise. J Comput Neurosci. 2011;30:759-78.

47. Peterka RJ. Postural control model interpretation of stabilogram diffusion analysis. Biol Cybern. 2000:82:335-43.

48. Peterka RJ. Comparison of human and humanoid robot control of upright stance. J Physiol Paris. 2009;103:149-58.

49. Horak FB, Nashner LM. Central programming of postural movements: adaptation to altered support-surface configurations. J Neurophysiol. 1986; 55:1369-81

50. Horak FB, Diener HC, Nashner LM. Influence of central set on human postural responses. J Neurophysiol. 1989;62:841-53.

51. Mok NW, Brauer SG, Hodges PW. Hip Strategy for Balance Control in Quiet Standing Is Reduced in People With Low Back Pain. Spine. 2004;29:E107-12. 
52. Fritz JM, Delitto A, Welch WC, Erhard RE. Lumbar spinal stenosis: a review of current concepts in evaluation, management, and outcome measurements. Arch Phys Med Rehabil. 1998;79:700-8.

53. Berg KO, Maki BE, Williams JI, Holliday PJ, Wood-Dauphinee SL. Clinical and laboratory measures of postural balance in an elderly population. Arch Phys Med Rehabil. 1992;73:1073-80.

54. Lee CE, Simmonds MJ, Novy DM, Jones S. Self-reports and clinicianmeasured physical function among patients with low back pain: a comparison. Arch Phys Med Rehabil. 2001;82:227-31.

Ready to submit your research? Choose BMC and benefit from:

- fast, convenient online submission

- thorough peer review by experienced researchers in your field

- rapid publication on acceptance

- support for research data, including large and complex data types

- gold Open Access which fosters wider collaboration and increased citations

- maximum visibility for your research: over $100 \mathrm{M}$ website views per year

At $\mathrm{BMC}$, research is always in progress.

Learn more biomedcentral.com/submissions 